\title{
Swelling Behavior of Partially Cross-Linked Polymers: A Ternary System
}

\author{
Souvik Nandi and H. Henning Winter* \\ Department of Chemical Engineering, University of Massachusetts-Amherst, \\ Amherst, Massachusetts 01003 \\ Received August 12, 2004; Revised Manuscript Received March 17, 2005
}

\begin{abstract}
Cross-linked polymer networks, irrespective of the method of cross-linking, contain a fraction of unattached polymer (sol macromolecules). During swelling in a solvent of low molecular weight, the sol macromolecules participate in the swelling process as an athermal solvent. Also, sol macromolecules begin to diffuse out of the swollen polymer into the surrounding solvent bath until equilibrium is reached between the sol macromolecules within the polymer specimen and the molecules in the surrounding bath. Thus, a partially cross-linked polymer during swelling should be treated as a ternary system consisting of polymer network, a high molecular weight solvent, and a low molecular weight solvent. On the basis of the Flory-Rehner theory, a first model has been developed to predict the swelling behavior of such ternary system. Transient states are considered as quasi-steady state, and the final condition of equilibrium is evaluated. The effect of polymer molecules in the surrounding solvent bath on the swelling behavior was also studied. Swelling experiments of partially cross-linked high-density polyethylene in $p$-xylene were performed to evaluate the model predictions. The presence of sol macromolecules within the swollen gel increased the swelling while sol molecules in the surrounding bath decreased the swelling of the partially cross-linked polymer.
\end{abstract}

\section{Introduction}

A chemically cross-linked polymer is capable of increasing its volume severalfold by absorbing large amounts of solvent. The swollen polymer network is held together by molecular strands that are connected by chemical bonds (cross-links). There are many different ways of cross-linking: irradiation using ultraviolet rays, X-ray photons, ions, electron-beam or $\alpha$-particles, or due to a chemical cross-linker. Irrespective of the method of cross-linking, some fraction of the polymer remains unattached to the network and/or gets detached by the cross-linking process (e.g., chain scission during radiation). The network content of the polymer is called the "gel fraction", and the fraction of loose macromolecules (sol macromolecules) is called the "sol fraction". In some cases, this sol fraction is large and its influence becomes important. During swelling of such partially cross-linked network in a low molecular weight solvent, the sol macromolecules act as an athermal solvent and interact entropically with the network. The sol macromolecules also tend to swell in the presence of the solvent ("solvent" henceforth will refer to as a low molecular weight solvent unless otherwise specified) and go into solution. Here we consider a system that is ternary in nature. It consists of network polymer, of sol macromolecules, and of solvent that diffuses into the polymer sample from the surface.

Typically, sol macromolecules are widely distributed in size and in architecture for several reasons. The initial polymer molecules, before cross-linking, are already distributed in size. Some of these molecules will remain without any change, while more of these will assemble in larger molecular clusters that are still mobile. Larger macromolecules are more likely to connect into the network than small macromolecules. In

\footnotetext{
* Corresponding author: $\mathrm{Ph}$ (413) 545-0922; Fax (413) 545-1647; e-mail winter@ecs.umass.edu.
}

the case of radiation cross-linking, chain scission will alter the macromolecular structure and also generate new macromolecules. A detailed account of these phenomena exceeds the scope of this study. In the following, sol macromolecules will be treated as a uniform species in a uniformly cross-linked matrix. All cross-links are assumed to be tetrafunctional.

During swelling, sol macromolecules diffuse out of the sample into the surrounding bath of solvent. The network would collapse if the volume, that previously was occupied by the lost sol macromolecules, would not be replenished by solvent molecules. A small amount of solvent is required to return the network to its stressfree state. Because of this phenomenon, we will use the original size of the polymer specimen (before exposure to solvent) as the reference size in the swelling study below. Swelling beyond the stress-free state contributes toward the elastic free energy of the swollen polymer.

The surrounding bath turns into a polymer solution due to sol macromolecules that diffuse out of the polymer sample. It will be interesting to see how the swelling behavior is affected by the presence of polymer molecules in the surrounding bath.

There are applications for such ternary systems. An example is the process of crystallization from swollen cross-linked gels (CSX) for making open-pore polymeric material, which uses a system that is ternary in nature. Partially cross-linked polyethylene or polypropylene is swollen in supercritical propane and then crystallized to obtain the open porous structure. The details of the CSX process are explained in our previous papers. ${ }^{1,2}$ Open-pore polymeric materials find a wide range application including superabsorbants, catalysis support, reaction media, and, in particular, separations such as filtration, pervaporation, gas separation, dialysis, and reverse osmosis. There is also a potential application of porous materials in medicine. Large pore size $(0.1-$ $100 \mu \mathrm{m})$ materials can be used as a scaffold for cell growth whereas small pore size $(\sim 10 \mathrm{~nm})$ materials can 
be used in membranes for making immuno-protective devices.

Hydrogels in biomedical application often involve ternary systems, the low molecular weight solvent being water. Silicone hydrogels are used in contact lenses; polyacrylamide hydrogels are used as absorbents in wound dressing; poly(vinyl alcohol) hydrogels are used for drug delivery applications and soft tissue replacements. The diffusion of the loose macromolecules in the swollen state plays an important role in these applications. ${ }^{31,32}$

The swelling of polymeric networks by a single solvent has been studied extensively. The studies concern the interaction of two components: a network polymer and a solvent. Several theories and modifications to existing theories have been presented to explain the swelling phenomena. Most of these studies concern the swelling of networks in a low molecular weight solvent while more recent studies allow the solvent to be high molecular weight. The classical theories for the swelling behavior of polymer networks in low molecular weight solvents go back to Flory and Wall ${ }^{3-7}$ and James and Guth. ${ }^{8,9}$ In the Wall-Flory-Rehner model, also known as the "affine network model", the movement of the network junctions is restricted. The microscopic deformation between any two points in the network is assumed to be proportional to the macroscopic deformation of the specimen. In the James-Guth model, usually known as the "phantom model", the cross-links in the bulk of the network fluctuate around their average positions. The magnitude of these fluctuations determines how strongly the macroscopic dilation of the network is coupled to the deformation of individual network strands. It is still debatable as to which of the theories is better at explaining the swelling behavior of polymer networks. Predictions from the phantom model are probably more realistic because the network junctions are allowed greater freedom of motion than in the affine model, but there are various reasons for the continued use of the Flory model. The model is simple and, thus, often used to estimate the actual degree of cross-linking in the network from its total swelling in a well-defined experiment. Many further theoretical models of rubber elasticity have been developed. However, most of the models have failed in describing the network behavior observed in experiments. Gottlieb and Gaylord ${ }^{10}$ used swelling data to compare different models and found that only a few of the theoretical model predictions agree qualitatively with the experimental data.

In this study, the classical theory of Wall, Flory, and Rehner for binary systems has been extended to accommodate the contributions of the sol macromolecules toward swelling. During swelling, two simultaneous diffusion effects need to be considered. The low molecular weight solvent molecules diffuse into the network from the sample surface while some of the sol macromolecules gradually diffuse out of the sample into the surrounding solvent. Effectively, the gel fraction of the polymer increases with time and potentially approaches $\phi_{\text {gel }} \rightarrow 1$ as most of the sol macromolecules get extracted.

\section{Background: Swelling of Binary Systems}

Flory-Rehner Model. The Flory-Rehner theory ${ }^{11}$ assumes linear superposition of the free energy changes associated with the swelling of the network, the free energy of mixing $\Delta F_{\mathrm{M}}$, and the elastic free energy $\Delta F_{\mathrm{el}}$ :

$$
\Delta F=\Delta F_{\mathrm{M}}+\Delta F_{\mathrm{el}}
$$

The additive nature of the free energies has been questioned in the past. Swelling experiments on poly(dimethylsiloxane) networks using different solvents, performed by Neuburger and Eichinger ${ }^{12}$ and later by Zhao and Eichinger, ${ }^{13}$ show a peak in dilation modulus $G^{\mathrm{d}}$ at an intermediate swelling ratio. This behavior conflicts with the Flory-Rehner theory that predicts $G^{\mathrm{d}}$ to be independent of the solvent. However, McKenna et al. ${ }^{14}$ performed a series of experiments with poly(isoprene) at different cross-link densities and temperatures and showed that the behavior of the "swelling activity parameter" $S$ (same as the dilation modulus $G^{\text {d }}$ used by Eichinger et al.) is ambiguous. The appearance of a maximum in a narrow range of temperature, and its disappearance beyond that cannot be explained currently. All the experiments discussed have been performed in limited amounts of solvent. At large degrees of swelling, the Flory-Rehner model, along with a few modifications, seems to provide satisfactory results. ${ }^{15}$

Experiments of McKenna et al. ${ }^{16}$ show that, except at high cross-link densities, the elastic free energy function of a dry polymer network is identical to that of the swollen polymer network although corrections need to be made for the polymer volume fraction in the swollen gel. Also, real polymer networks contain macromolecular branches ("dangling ends") and various imperfections that may not contribute to the permanent network elasticity. This results in a reduced elastic osmotic pressure and increased swelling. Flory ${ }^{17-19}$ modified the original affine model to rationalize the discrepancies between the basic model and the experimental observations. After the modification of the classical rubber elasticity theory, ${ }^{11}$ the elastic free energy for a swollen network with tetrafunctional junction point was represented as

$$
\frac{\Delta F_{\mathrm{el}}}{k_{\mathrm{b}} T}=\kappa\left(V_{\mathrm{P}} v_{\mathrm{e}} / 2\right) \sum_{i=1}^{3}\left(\alpha_{i}^{2}-1-\ln \alpha_{i}\right)
$$

where $\alpha_{i}$ is the linear deformation factor, $\left(V / V_{\mathrm{P}}\right)^{1 / 3}, V_{\mathrm{P}}$ is the volume of dry polymer, $V$ is the volume of the swollen polymer, $k_{\mathrm{b}}$ is the Boltzmann constant, and $T$ is the absolute temperature. The fraction of active network $\kappa(\leq 1)$ is a fitting parameter that accounts for network imperfections, and $v_{\mathrm{e}}$ is the cross-link density per unit polymer volume. The average molecular weight of the strands between cross-links $M_{\mathrm{C}}$ can be related to the cross-link density as $v_{\mathrm{e}}=\rho_{\mathrm{P}} / M_{\mathrm{C}}$, where $\rho_{\mathrm{P}}$ is the density of the bulk polymer.

The mixing free energy term includes enthalpic and the entropic contributions of the solvent molecules and the network. ${ }^{20,21}$ The mixing free energy $\Delta F_{\mathrm{m}}$ can be written as

$$
\frac{\Delta F_{\mathrm{m}}}{k_{\mathrm{b}} T}=\left[n_{\mathrm{S}} \ln \left(1-v_{\mathrm{N}}\right)+\chi n_{\mathrm{S}} v_{\mathrm{N}}\right]
$$

where $n_{\mathrm{S}}$ is the number of solvent molecules, $\chi$ is the Flory-Huggins enthalpic interaction parameter of the solvent and the network, and $v_{\mathrm{N}}$ is the volume fraction of the polymer network in the swollen gel. For an isotropic network, where the swelling is independent of the orientation of the polymer, $\alpha_{1}=\alpha_{2}=\alpha_{3}=\alpha$. When rearranging these equations, it will be assumed that the 
linear deformation factors $\alpha_{i}$ are related to the polymer volume fraction in a swollen gel as $\alpha_{1} \alpha_{2} \alpha_{3}=V / V_{\mathrm{P}}=1 / v_{\mathrm{N}}$.

The total free energy changes associated with the swelling of a polymer network obtained by the addition of eqs 2 and 3 can be written as

$$
\begin{aligned}
\frac{\Delta F}{k_{\mathrm{b}} T}=\left[n_{\mathrm{S}} \ln \left(1-v_{\mathrm{N}}\right)+\right. & \left.\chi n_{\mathrm{S}} v_{\mathrm{N}}\right]+ \\
& \kappa\left(V_{\mathrm{P}} v_{\mathrm{e}} / 2\right)\left(3 \alpha^{2}-3-\ln \alpha^{3}\right)
\end{aligned}
$$

The equilibrium state is defined by the equivalence of the chemical potential of the solvent molecules inside the swollen gel and in the surrounding fluid. $v_{\mathrm{e}}$ and $\alpha$ in eq 4 can be replaced by the corresponding definitions and the chemical potential can be calculated from the total free energy. For an isotropic network, the swelling equilibrium of a polymer network in a solvent can now be represented as

$$
\begin{array}{r}
\mu_{\mathrm{S}}-\mu_{\mathrm{S}}^{\mathrm{o}}=\frac{\partial}{\partial n_{\mathrm{S}}}\left(\frac{\Delta F}{k_{\mathrm{b}} T}\right)_{T, P}=\left[\ln \left(1-v_{\mathrm{N}}\right)+v_{\mathrm{N}}+\chi v_{\mathrm{N}}^{2}\right]+ \\
\kappa \rho_{\mathrm{P}} \tilde{V}_{\mathrm{S}} M_{\mathrm{c}}^{-1}\left(v_{\mathrm{N}}^{1 / 3}-v_{\mathrm{N}} / 2\right)=0
\end{array}
$$

where $\tilde{V}_{\mathrm{S}}$ is the molar volume of the solvent. The $\chi$ parameter was observed to change linearly with the cross-link density of the network. ${ }^{22}$

Swelling of Network Polymer in High Molecular Weight Solvent. Gent and Tobias ${ }^{23}$ obtained large diffusion coefficients for linear PDMS molecules diffusing into PDMS networks. The diffusion into the network was observed to resemble self-diffusion. The equilibrium swelling experimental data were in good agreement with the predictions from the Flory-Huggins theory of swelling with a zero heat of interaction $(\chi=0)$ between the network PDMS and the linear PDMS.

Russ et al. ${ }^{24}$ extended the Flory-Rehner model to solvents of high molecular weight. Polystyrene networks were swollen in linear polystyrene molecules of increasing molecular weights. It was observed that both the affine and the phantom model with a few modifications could predict the swelling behavior of the network. Similar to swelling of networks with low molecular weight solvents, ${ }^{24}$ the $\chi$ parameter was found to depend on the cross-link density for the swelling of networks with polymer molecules. However, the value of $\chi$ obtained from the experiments was independent of the molecular weight of the linear polymer. Unlike Neuburger and Eichinger, ${ }^{12}$ who obtained a peak in the dilation modulus at a certain swelling ratio, no peak was observed in the swelling of a network in polymeric solvent. This interesting observation suggests that the elastic free energy is independent of the molecular weight of the macromolecular solvent.

\section{Swelling Behavior of Ternary Systems in Polymer Solution}

Enthalpic and Entropic Interactions. In a most basic attempt of modeling the ternary system, we start out with the Flory-Rehner model (eq 1). Additional contributions account for the interaction of the loose macromolecules (sol fraction) with the polymer network and with the low molecular weight solvent. The sol macromolecules, in addition to the solvent molecules, contribute toward the entropic free energy of mixing analogous to the Flory-Huggins type of behavior of polymer solutions. The enthalpic interactions of the

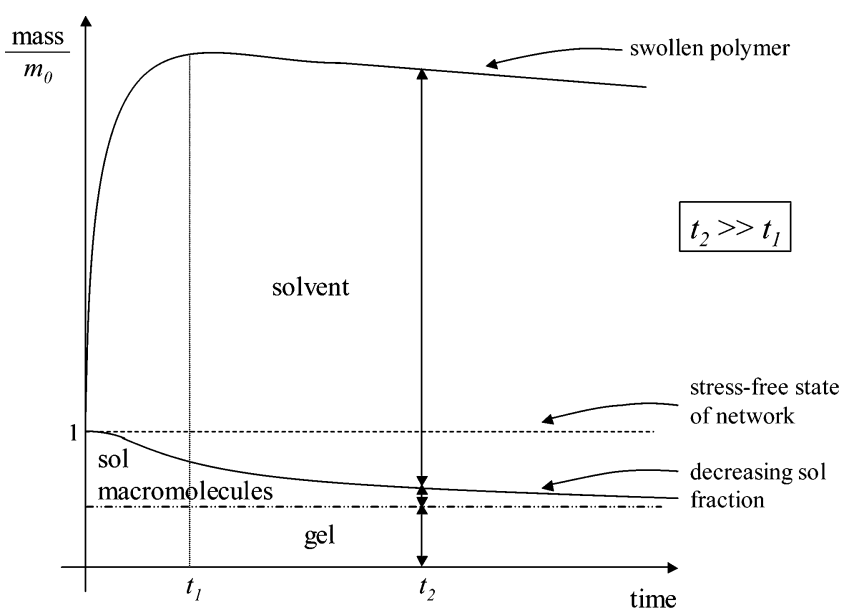

Figure 1. Time-dependent mass of a partially cross-linked polymer sample during swelling in a low molecular weight solvent. Solvent molecules diffuse into the polymer within a short time and swell the polymer. Sol macromolecules are lost from the swollen gel over a long time. Fractions of solvent molecules swelling the polymer replace the lost sol macromolecules to bring the network back to its stress-free state. The network when swollen beyond the stress-free state contributes toward the elastic free energy.

solvent molecules with the network and the sol fraction are included. The interaction parameter $\chi$ between the network strands and the solvent has been reported to depend on the cross-link density. ${ }^{22,24}$ It is possible that the value of $\chi$ is different for the network and the sol macromolecules. For the system considered here, the molecular structures of the network and the sol macromolecules are the same, and any difference in the value of the interaction parameter is neglected. On the basis of the same reasoning, the interaction between the network and the sol macromolecules is neglected. The free energy of mixing is then represented as

$$
\frac{\Delta F_{\mathrm{m}}}{k_{\mathrm{b}} T}=n_{\mathrm{S}} \ln v_{\mathrm{S}}+n_{\mathrm{L}} \ln v_{\mathrm{L}}+\chi n_{\mathrm{S}}\left(v_{\mathrm{L}}+v_{\mathrm{N}}\right)
$$

where subscript $\mathrm{S}$ stands for the small molecular weight solvent, L stands for the large molecular weight solvent (sol macromolecules), and $\mathrm{N}$ stands for the network. It is to be noted that $\left(v_{\mathrm{L}}+v_{\mathrm{N}}\right)$ is the total volume fraction of polymer $v_{\mathrm{P}}$ in the swollen gel. The additional terms arise from the entropic and enthalpic interaction of the sol macromolecules.

Elastic Contributions. The sol macromolecules do not contribute toward the elastic free energy. Only the network participates in the retractable force during swelling. Therefore, the cross-link density $v_{\mathrm{e}}$ is defined with respect to the network fraction of the polymer, unlike the original Flory model, where the network is comprised of the entire polymer. The volume of the network is obtained as $V_{\mathrm{N}}=\phi_{\text {gel }} V_{\mathrm{P}}$, where $\phi_{\text {gel }}$ is the gel fraction. However, during swelling, the potential gradient across the swollen polymer drives the loose chains into the bulk solvent. This phenomenon reduces $V_{\mathrm{P}}$ and increases the gel fraction of the polymer. The mass of the network remains unchanged (Figure 1). At all times during the swelling of the network, the crosslink density is represented by

$$
v_{\mathrm{e}}=\frac{\rho_{\mathrm{P}} \phi_{\text {gel }}}{M_{\mathrm{C}}}
$$

where $\phi_{\text {gel }}$ varies with time. 
When the network is formed by the cross-linking reaction, certain volume is occupied by the sol macromolecules. During swelling, as more and more sol macromolecules leave the polymer, the network contracts. The strain developed in the network strands results in a stress. Fractions of solvent molecules swelling the polymer have to replace the volume occupied by the lost sol macromolecules to bring the network back to its stress-free state (Figure 1). Any swelling of the polymer beyond this stress-free state will generate an elongation of the network strands. Thus, the elastic contributions of the network appear only with respect to the stress-free state of the network. The linear deformation factor $\alpha$ defines the increase in volume of the polymer from its stress-free state

$$
\alpha^{3}=\frac{V_{\mathrm{SP}}}{V_{\mathrm{P}}^{\mathrm{i}}}=\left(\frac{\phi_{\text {gel }}^{\mathrm{i}}}{\phi_{\text {gel }}}\right) \frac{1}{v_{\mathrm{P}}}
$$

where $V_{\mathrm{SP}}$ is the volume of the swollen polymer, $V_{\mathrm{P}}^{\mathrm{i}}$ is the volume of the initial stress-free partially cross-linked polymer, and $\phi_{\text {gel }}^{\mathrm{i}}$ is the initial gel fraction. If the volume of the swollen polymer is large compared to the volume of the sol macromolecules lost during swelling, $\alpha^{3}$ can be approximated as the ratio of the volume of the swollen polymer $V_{\mathrm{SP}}$ to the volume of the dry polymer $V_{\mathrm{P}}: \alpha^{3}=1 / v_{\mathrm{P}}$.

Considering isotropic swelling and the junctions being tetrafunctional, eq 2 can be reduced to obtain the elastic free energy of the network as

$$
\frac{\Delta F_{\mathrm{el}}}{k_{\mathrm{b}} T}=\kappa^{\prime}\left(V_{\mathrm{P}} v_{\mathrm{e}} / 2\right)\left(3 \alpha^{2}-3-\ln \alpha^{3}\right)
$$

where $v_{\mathrm{e}}$ is as defined above.

The above model can be extended to include constraints that are imposed upon network junctions by their surrounding network strands. The constraint reduces the average number of degrees of freedom of a network strand. The "localization model" of Gaylord and Douglas $^{25}$ concentrates on this effect. When a dry polymer network undergoes dilation, the cooperative motion of the chains contributes to the elastic free energy in addition to the connectivity of the chains (classical rubber theory). These restrictions on the network chains increase the free energy by

$$
\Delta F_{\text {loc }}=3 G_{\text {loc }} V_{\mathrm{P}}(\lambda-1)
$$

where $G_{\text {loc }}$ is the localization modulus and $\lambda$ is the principal extension ratio. The free energy increase due to the cooperative motion of the chains is independent of the nature of the deformation, be it shear or linear elongation or swelling in a solvent. In the case of isotropic swelling, the principal extension ratio $\lambda$ is identical to the linear deformation factor $\alpha$. The localization modulus has two contributions

$$
G_{\mathrm{loc}}=\gamma\left(k_{\mathrm{b}} T v_{\mathrm{e}} / 2\right)+G_{\mathrm{e}}
$$

where $\gamma$ is a constant and $G_{\mathrm{e}}$ is the equilibrium modulus of the un-cross-linked polymer melt. $G_{\mathrm{e}}$ remains constant for a polymer network undergoing extension in absence of any solvent. However, for a swollen polymer, the value of the equilibrium modulus decreases due to solvent uptake. When the polymer swells by a factor $\alpha$, Douglas and McKenna ${ }^{26}$ estimated $G_{\mathrm{e}}$ to decrease as

$$
G_{\mathrm{e}}(\alpha)=G_{\mathrm{e}} v_{\mathrm{N}}=G_{\mathrm{e}} \phi_{\mathrm{gel}} \alpha^{-3}
$$

Inserting the above definition of the equilibrium modulus into eq 11 and combining it with eqs 9 and 10, the elastic free energy of a polymer network becomes

$$
\begin{aligned}
& \frac{\Delta F_{\mathrm{el}}}{k_{\mathrm{b}} T}=\kappa\left(V_{\mathrm{P}} v_{\mathrm{e}} / 2\right)\left(3 \alpha^{2}+3 \alpha-6\right.\left.-\ln \alpha^{3}\right)+ \\
& 3 \frac{V_{\mathrm{P}}}{k_{\mathrm{b}} T} \frac{G_{\mathrm{e}} \phi_{\text {gel }}}{\alpha^{3}}(\alpha-1)
\end{aligned}
$$

The total free energy of the swollen gel is given by the linear superposition of eqs 6 and 13 .

$$
\begin{array}{r}
\frac{\Delta F}{k_{\mathrm{b}} T}=n_{\mathrm{S}} \ln v_{\mathrm{S}}+n_{\mathrm{L}} \ln v_{\mathrm{L}}+\chi n_{\mathrm{S}}\left(v_{\mathrm{L}}+v_{\mathrm{N}}\right)+ \\
\kappa\left(V_{\mathrm{P}} v_{\mathrm{e}} / 2\right)\left(3 \alpha^{2}+3 \alpha-6-\ln \alpha^{3}\right)+ \\
3 \frac{V_{\mathrm{P}}}{k_{\mathrm{b}} T} \frac{G_{\mathrm{e}} \phi_{\text {gel }}}{\alpha^{3}}(\alpha-1)
\end{array}
$$

Macromolecules in the Solvent Bath. The free energy of the surrounding solution can be treated as the Flory-Huggins free energy of mixing of a polymer solution:

$\frac{\Delta F^{\text {surr }}}{k_{\mathrm{b}} T}=n_{\mathrm{S}}^{\text {surr }} \ln v_{\mathrm{S}}^{\text {surr }}+n_{\mathrm{L}}^{\text {surr }} \ln v_{\mathrm{L}}^{\text {surr }}+\chi n_{\mathrm{S}}^{\text {surr }} v_{\mathrm{L}}^{\text {surr }}$

The superscript "surr" stands for the polymer solution that surrounds the specimen. The other symbols stand for the usual notation. The volume fraction of polymer in the surrounding solution, $v_{\mathrm{L}}^{\text {surr }}$, is treated as a free parameter. It can attain different values depending on the swelling environment. A predetermined concentration of polymer solution can be used in the bath for the swelling experiments (see Experimental Section). In such a situation, the loss of sol macromolecules from the swollen gel adds to the volume fraction of the polymer in the surrounding solution. The limiting case, when swelling begins in a pure solvent, will be discussed later. It is to be noted that the presence of macromolecules in the surrounding solution cannot be avoided during swelling of partially cross-linked polymers, but it can be kept small by using a large bath/sample ratio. Polymer molecules dissolved in the solvent surrounding the gel will affect the chemical potential and, hence, the degree of swelling of the network.

Combination of Terms. During swelling, the diffusion of the solvent into the gel is assumed to be much faster than the diffusion of sol macromolecules out of the gel. Such assumption is considered reasonable because the solvent molecules are much smaller than the sol macromolecules. After the initial swelling period, the swollen gel is considered to be at a quasi-steady state at any instant of time.

A quasi-steady state for the ternary system is reached when the chemical potential of the solvent molecules in the gel equates the chemical potential of the solvent molecules in the surrounding solution.

$$
\begin{aligned}
& \mu_{\mathrm{S}}-\mu_{\mathrm{S}}^{\mathrm{surr}}=\left(\mu_{\mathrm{S}}-\mu_{\mathrm{S}}^{o}\right)-\left(\mu_{\mathrm{S}}^{\mathrm{surr}}-\mu_{\mathrm{S}}^{o}\right)= \\
& \left(\frac{\partial\left(\Delta F / k_{\mathrm{b}} T\right)}{\partial n_{\mathrm{S}}}\right)_{T, P}-\left(\frac{\partial\left(\Delta F^{\mathrm{surr}} / k_{\mathrm{b}} T\right)}{\partial n_{\mathrm{S}}^{\mathrm{surr}}}\right)_{T, P}=0
\end{aligned}
$$


The free energy terms (eqs 14 and 15) are used in eq 16 a to obtain the ternary model.

$$
\begin{aligned}
& \ln \left(1-v_{\mathrm{P}}\right)+v_{\mathrm{P}}-\frac{1}{x} v_{\mathrm{P}}\left(1-\phi_{\text {gel }}\right)+\chi\left(v_{\mathrm{P}}\right)^{2}+ \\
& \kappa \frac{\rho \tilde{V}_{\mathrm{S}} \phi_{\text {gel }}^{\mathrm{i}}}{M_{\mathrm{c}}}\left(\left(\frac{\phi_{\text {gel }}}{\phi_{\text {gel }}^{\mathrm{i}}} v_{\mathrm{P}}\right)^{1 / 3}+\frac{1}{2}\left(\frac{\phi_{\text {gel }}}{\phi_{\text {gel }}^{\mathrm{i}}} v_{\mathrm{P}}\right)^{2 / 3}-\frac{1}{2}\left(\frac{\phi_{\text {gel }}}{\phi_{\text {gel }}^{\mathrm{i}}} v_{\mathrm{P}}\right)\right)+ \\
& \frac{G_{\mathrm{e}} \tilde{V}_{\mathrm{S}} \phi_{\text {gel }}^{\mathrm{i}}\left(\frac{\phi_{\text {gel }}}{R T} v_{\mathrm{P}}\right)^{5 / 3}\left(3\left(\frac{\phi_{\text {gel }}}{\phi_{\text {gel }}^{\mathrm{i}}} v_{\mathrm{P}}\right)^{1 / 3}-2\right)=}{\ln \left(1-v_{\mathrm{L}}^{\text {surr }}\right)+\left(1-\frac{1}{x}\right) v_{\mathrm{L}}^{\text {surr }}+\chi\left(v_{\mathrm{L}}^{\text {surr }}\right)^{2}}(16 \mathrm{~b})
\end{aligned}
$$

In the above equation, $x$ is the ratio of the molar volume of the sol macromolecules to the molar volume of the low molecular weight solvent and $\phi_{\text {gel }}$ is the gel fraction of the polymer at the instant of time when the swelling is measured. For the Flory-Huggins lattice model, $x$ is equal to the degree of polymerization of the sol macromolecules.

It is most informative to compare the ternary model to the classical Flory-Rehner model for binary systems. We start with the enthalpic term containing the interaction parameter $\chi$. The model for the ternary system consists of two additional terms $\left(\chi\left(v_{\mathrm{N}}+v_{\mathrm{L}}\right)^{2}-\chi\left(v_{\mathrm{L}}^{\text {surr }}\right)^{2}\right)$ when compared to the binary model $\left(\chi v_{\mathrm{N}}^{2}\right)$. This implies that the sol macromolecules inside the swollen gel increase the swelling while any polymer molecules in the surrounding solution reduce the swelling. The entropic contributions of the sol macromolecules appear as an additional term $v_{\mathrm{P}}\left(1-\phi_{\mathrm{gel}}\right) / x$ for the ternary system. This contribution is proportional to the volume fraction of the sol macromolecules $v_{\mathrm{L}}$. Thus, the entropic contributions of the sol macromolecules decrease over time as they diffuse out of the swollen gel. Several new terms enter the equation in a nonlinear way. They are not simply additive because of the three-way interactions between the components.

Equilibrium State. Equilibrium is reached when the chemical potential of the sol macromolecules within the gel becomes equal to the chemical potential of the loose chains in the surrounding solution.

$$
\begin{aligned}
& \mu_{\mathrm{L}}-\mu_{\mathrm{L}}^{\text {surr }}=\left(\mu_{\mathrm{L}}-\mu_{\mathrm{L}}^{0}\right)-\left(\mu_{\mathrm{L}}^{\text {surr }}-\mu_{\mathrm{L}}^{0}\right)= \\
& \left(\frac{\partial\left(\Delta F / k_{\mathrm{b}} T\right)}{\partial n_{\mathrm{L}}}\right)_{T, P}-\left(\frac{\partial\left(\Delta F^{\text {surr }} / k_{\mathrm{b}} T\right)}{\partial n_{\mathrm{L}}^{\text {surr }}}\right)_{T, P}=0
\end{aligned}
$$

When swelling of the polymer begins in a pure solvent, final equilibrium is reached after a long time when most of the sol macromolecules have left the gel ( $\phi_{\text {gel }} \rightarrow 1$ for a solvent bath of infinite size). However, if the solvent bath is small and contains polymer molecules from the beginning, chemical potential of the polymer chains in the surrounding solution will increase as the sol macromolecules diffuse out. The difference between the chemical potential of the sol macromolecules within the swollen gel and in the surrounding solution $\left(\mu_{\mathrm{L}}-\mu_{\mathrm{L}}^{\text {surr }}\right)$ will decrease rapidly. Equation $17 \mathrm{a}$ can be simplified as

$$
\begin{aligned}
& \ln \left[\left(1-\phi_{\text {gel }}\right) v_{\mathrm{P}}\right]+\left(1-v_{\mathrm{P}}\right)\left(1-x+\chi x\left(1-v_{\mathrm{P}}\right)\right)+ \\
& \frac{\kappa \rho_{\mathrm{P}} \phi_{\text {gel }}^{\mathrm{i}} x \tilde{V}_{\mathrm{S}}}{M_{\mathrm{C}}}\left(\left(\frac{\phi_{\text {gel }}}{\phi_{\text {gel }}^{\mathrm{i}}} v_{\mathrm{P}}\right)^{1 / 3}+\frac{1}{2}\left(\frac{\phi_{\text {gel }}}{\phi_{\text {gel }}^{\mathrm{i}}} v_{\mathrm{P}}\right)^{2 / 3}-\frac{1}{2}\left(\frac{\phi_{\text {gel }}}{\phi_{\text {gel }}^{\mathrm{i}}} v_{\mathrm{P}}\right)\right)- \\
& \frac{G_{\mathrm{e}} \tilde{V}_{\mathrm{S}} \phi_{\text {gel }}^{\mathrm{i}} x}{k_{\mathrm{b}} T}\left(\frac{\phi_{\text {gel }}}{\phi_{\text {gel }}^{\mathrm{i}}} v_{\mathrm{P}}\right)\left(2\left(\frac{\phi_{\text {gel }}}{\phi_{\text {gel }}^{\mathrm{i}}} v_{\mathrm{P}}\right)^{2 / 3}-3\left(\frac{\phi_{\text {gel }}}{\phi_{\text {gel }}^{\mathrm{i}}} v_{\mathrm{P}}\right)\right)= \\
& \ln v_{\mathrm{L}}^{\text {surr }}+(1-x)\left(1-v_{\mathrm{L}}^{\text {surr }}\right)+\chi x\left(1-v_{\mathrm{L}}^{\text {surr }}\right)^{2}
\end{aligned}
$$

Equation 16b characterize the equivalence of chemical potential of the solvent molecules inside the swollen gel and in the surrounding solution while eq $17 \mathrm{~b}$ characterizes the equivalence of the chemical potential of sol macromolecules within the swollen gel and in the surrounding solution. At the equilibrium state, the chemical potentials of both the solvent molecules and the sol macromolecules satisfy the equivalence criteria. Therefore, the gel fraction and the degree of swelling at the final equilibrium state obtained from eq $17 \mathrm{~b}$ will also be satisfied by eq $16 \mathrm{~b}$. Hence, this can be used to estimate physical parameters such as the interaction parameter $\chi$, which is otherwise difficult to determine.

\section{Experimental Section}

Materials. Industrial grade cross-linked high-density polyethylene (HDPE) was used to perform the swelling experiments. The high-density polyethylene was cross-linked by electron radiation at three different radiation doses of 5, 7.5, and $10 \mathrm{Mrad}$. The samples were partially cross-linked with a fraction of sol macromolecules remaining within the sample. 99\% p-xylene (Aldrich Chemical Co.) was used as the low molecular weight solvent for all the experiments. 2-Ethyl-1hexanol (Aldrich Chemical Co.) was used to extract the solvent from the swollen gel.

Determination of Gel Fraction. The gel fraction of the polymer was measured by an ASTM method. ${ }^{27}$ Around $100 \mathrm{mg}$ of each of the polymer samples was exposed to $p$-xylene at 100 ${ }^{\circ} \mathrm{C}$ for $72 \mathrm{~h}$, the solvent getting replaced with fresh $p$-xylene every $24 \mathrm{~h}$. This process extracted most of the loose chains from the network. The samples were then removed from the solvent and dried under vacuum at $80{ }^{\circ} \mathrm{C}$ for $24 \mathrm{~h}$. The gel fraction was calculated as the mass ratio of the dry sample to the initial sample.

Experimental Procedure. According to the theory for the partially cross-linked polymer networks (eq 7), the cross-link density per unit volume of polymer is expected to vary linearly with the gel fraction. Samples with varying gel fraction were prepared to determine the cross-link density. Cross-linked HDPE specimens $(14 \mathrm{~mm} \times 14 \mathrm{~mm} \times 1.3 \mathrm{~mm})$ were swollen in $p$-xylene at $100{ }^{\circ} \mathrm{C}$. During solvent exposure, fractions of sol macromolecules diffuse out of the specimen into the bath. This causes the gel fraction of the specimen to increase with time. To obtain different gel fractions, samples were removed from the solvent every $4 \mathrm{~h}$ and put in 2-ethyl-1-hexanol at 150 ${ }^{\circ} \mathrm{C}$ to extract $p$-xylene from the swollen gel. The remaining solvent was removed from the sample by applying vacuum for appropriate times. The dried samples were weighed, and the gel fraction was calculated from the ratio of their instantaneous mass to the initial mass.

The compression modulus of these samples at various gel fractions was measured in a linear rheometer (Rheometrics RDS-LA controlled by a Labview program that has been developed by Roland Horst). The samples were compressed at a constant strain rate of $0.005 \mathrm{~s}^{-1}$, and the resulting compression force was measured. Strain rate $\dot{\epsilon}$ and strain $\epsilon$ are defined with the sample height $H(t)$ :

$$
\dot{\epsilon}=\frac{\mathrm{d}(\ln H(t))}{\mathrm{d} t} \quad \epsilon=\ln \left(\frac{H(t)}{H_{0}}\right)
$$


Table 1. HDPE Samples with Different Radiation Doses ${ }^{a}$

\begin{tabular}{cccc}
\hline HDPE & initial gel fraction & $M_{\mathrm{c}}$ & $\kappa$ \\
\hline $5.0 \mathrm{MRad}$ & 0.12 & $1.5 \times 10^{4}$ & 0.37 \\
$7.5 \mathrm{MRad}$ & 0.28 & $1.1 \times 10^{4}$ & 0.25 \\
$10 \mathrm{MRad}$ & 0.41 & $7.3 \times 10^{3}$ & 0.21
\end{tabular}

${ }^{a}$ The initial gel fraction was determined by the ASTM method. The molecular weight of strands between cross-links was determined from the compression modulus experiments. $\kappa$ was determined by fitting the model for a ternary system to the experimental results (Figure 4).

Stress was calculated by dividing the measured normal force by the cross-sectional area of the rheometer disk fixtures. The compression modulus $E$ is defined as the slope of the stressstrain curve. Molecular weight of strands between cross-links $M_{\mathrm{C}}$ was estimated from $E$ (described below) and inserted in the model to predict the swelling behavior of the partially cross-linked networks.

Swelling experiments were performed with the cross-linked HDPE samples in $p$-xylene at $100{ }^{\circ} \mathrm{C}$. The primary objective behind the swelling experiments was to obtain the degree of swelling of each of the samples at increasing gel fraction. Samples were first swollen in a limited amount of $p$-xylene to minimize loss of sol fraction during swelling. $2 \mathrm{~g}$ of $p$-xylene was taken in a vial and heated to $100{ }^{\circ} \mathrm{C}$ in an oil bath. A specimen of initial mass $m^{\mathrm{i}}$ was put in the hot solvent, and the vial was sealed to prevent loss of solvent. After an initial swelling period of $18 \mathrm{~h}$ (which was observed as the time taken to reach the initial quasi-steady state), the contents of the vial were transferred to a flask containing $100 \mathrm{~mL}$ of $p$-xylene at the same temperature. The specimen was allowed to lose fractions of sol macromolecules in an excess of solvent. Several specimens of each sample were put through the same conditions. Specimen after specimen was removed sequentially from the solvent at increasing times. The fraction of sol macromolecules inside the specimen reduces with soak time. For the analysis, it is assumed that the rate of diffusion of the sol macromolecules inside the gel is same for all the specimens.

The removed specimens were put in a preweighed vial and sealed. The temperature was maintained constant while transferring the specimens from the flask into the vial. This was very important since the amount of solvent taken up by the polymer is very sensitive to temperature. The sealed vial was weighed and its difference from the mass of the empty vial provided the mass of the swollen gel. The specimens were then dried in a vacuum oven at $80^{\circ} \mathrm{C}$ for $24 \mathrm{~h}$ and weighed to obtain the mass $m$ left after loss of fractions of sol macromolecules. The gel fraction of each specimen was calculated from the initial gel fraction $\phi_{\text {gel }}^{\mathrm{i}}$ as

$$
\phi_{\text {gel }}=\phi_{\text {gel }}^{\mathrm{i}}\left(\frac{m^{\mathrm{i}}}{m}\right)
$$

The mass of the solvent taken up by the swollen gel was calculated as the difference of the mass of the swollen polymer and the dried polymer. The volume of the swollen gel was calculated from the mass of polymer and the mass of solvent taken up considering the densities of the amorphous polymer and $p$-xylene at the experimental temperatures. The ratio of the volume of the swollen polymer to that of the dry polymer is defined as the degree of swelling.

\section{Results and Discussion}

Experimental Observations. Table 1 lists the initial gel fraction of all the samples. The gel fraction increases with radiation dose. The low values of the gel fraction indicate that there is a substantial amount of un-cross-linked sol macromolecules present in the polymer that participate in the swelling.

Radiation damages soft matter. Also, radiation might result in a gradient in cross-linking due to the weakening of the radiation from one side of the sample to the other. To verify this effect, a $1.3 \mathrm{~mm}$ thick PE 10 sample was cut along the thickness of the polymer to obtain two $0.65 \mathrm{~mm}$ thick samples. The top half and the bottom half were both cut into smaller pieces to obtain a number of specimens. Each of these specimens was swollen in $p$-xylene. The degree of swelling was measured, and the corresponding gel fractions were calculated. It was observed that swelling of the top half was about $2.5 \%$ less than the bottom half. This small value implies that the difference in cross-link density over the thickness of the polymer can be neglected.

Figure 2 shows the stress-strain relationships of the three samples at different gel fraction. The compression modulus $E$ increases with increase in gel fraction for each of the three samples. The cross-link density of the network per unit volume of the polymer $v_{\mathrm{e}}$ is calculated from $E^{28}$

$$
v_{\mathrm{e}}=\frac{E}{3 R T}
$$

$v_{\mathrm{e}}$ calculated from $E$ for the three HDPE samples at different gel fractions were plotted against their corresponding $\phi_{\text {gel }}$ (Figure 3). The gel fraction increases as the sol macromolecules are partially extracted from the polymer while the mass of the network remains unchanged. This results in an increase in network density per unit volume of the polymer as observed in Figure 3. The molecular weight of strands between cross-links $M_{\mathrm{C}}$ can be obtained from the slopes of $v_{\mathrm{e}}$ vs $\phi_{\text {gel }}$ according to eq $7 . M_{\mathrm{C}}$ (Table 1) decreases with increase in radiation dose. These values of $M_{\mathrm{C}}$ were later used in the model (eqs $16 \mathrm{~b}$ and $17 \mathrm{~b}$ ).

The experimental results in Figure 4 show that the sol macromolecules increase the degree of swelling of the polymer. It is observed that a presence of $50 \%$ of loose chains increases the degree of swelling by $30-45 \%$ depending on the molecular weight between cross-links in the polymer. The sol macromolecules act as an athermal solvent in the system and increase swelling. The increase in network swelling due to the presence of sol macromolecules is quite significant and cannot be neglected.

Model Predictions. The values of the molecular weight of strands between cross-links determined from the compression modulus experiments were used in the model developed above to predict the volume fraction of polymer $v_{\mathrm{P}}$ during swelling of the partially crosslinked HDPE in $p$-xylene (Figure 4). In each of our experiments the polymer in the surrounding solvent originated only from the loss of sol macromolecules from the gel. No additional polymer was dissolved in the solvent prior to the swelling. Thus, in the limiting case of our ternary system, the polymer concentration in the surrounding solution $v_{\mathrm{L}}^{\text {surr }}$ can be determined from a mass balance equation

$$
v_{\mathrm{L}}^{\text {surr }}=\frac{V_{\mathrm{P}} \phi_{\text {gel }}}{V^{\text {surr }}}\left(\frac{1-\phi_{\text {gel }}^{\mathrm{i}}}{\phi_{\text {gel }}^{\mathrm{i}}}-\frac{1-\phi_{\text {gel }}}{\phi_{\text {gel }}}\right)
$$

where $V^{\text {surr }}$ is the volume of the surrounding solvent and $\phi_{\text {gel }}^{\mathrm{i}}$ is the initial gel fraction of the cross-linked polymer. $v_{\mathrm{L}}^{\text {surr }}(t)$ is a function of time but eventually reaches an equilibrium value. The other physical parameters used in the model are obtained from the literature. ${ }^{29,30}$ $\kappa$ was determined by fitting the model to the experimental results. The values of $\kappa$ are listed in Table 1. 

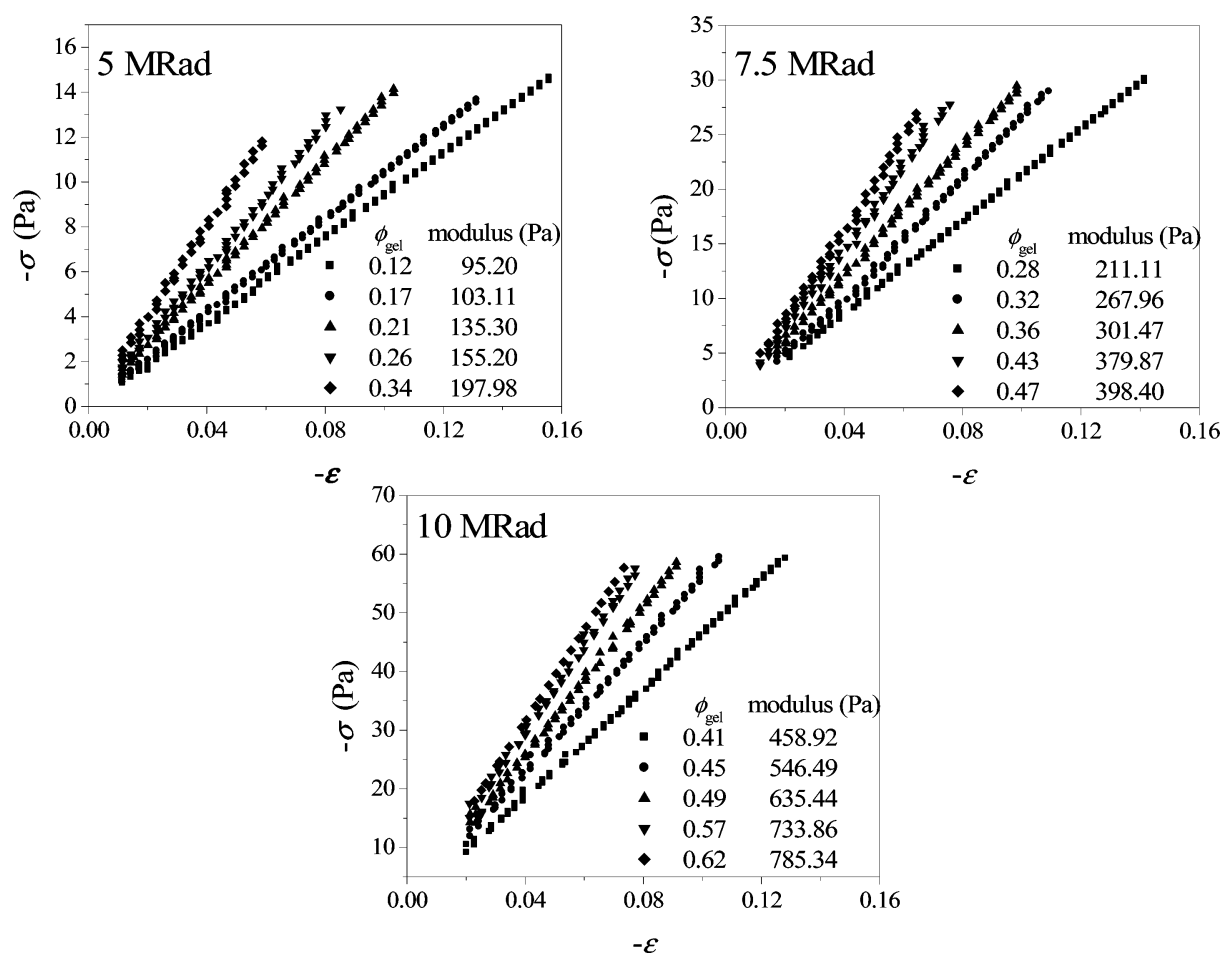

Figure 2. Compression modulus results for the three HDPE samples at different gel fractions. Experiments were performed at $170{ }^{\circ} \mathrm{C}$ and at a strain rate $(=\mathrm{d}(\ln H(t)) / \mathrm{d} t)$ of $0.005 \mathrm{~s}^{-1}$.

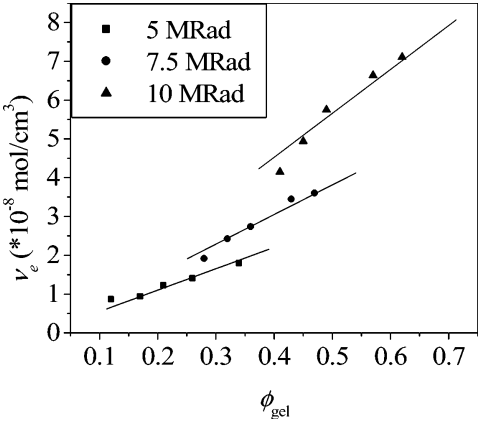

Figure 3. Cross-link density increases with increase in gel fraction. The molecular weight of strands between cross-links calculated from the slopes of the curves decreases with increase in radiation dose.

The low values of $\kappa$ indicate the presence of a large number of loose ends and network imperfections. This is in accordance with the low compression modulus values and the large $M_{\mathrm{C}}$ values.

It was interesting to see that the swelling of the polymer increases significantly due to the presence of sol macromolecules. The reason for the increase in swelling might be explained as follows. When the loose polymer chains are brought in contact with a good solvent, they tend to uncoil, expand, and go into solution, which is an entropically more favorable state. However, expansion is restricted by the presence of the network. This makes the loose molecules to exert a secondary stress on the network and, thus, causes it to swell more. As the loose molecules diffuse out of the polymer, the supplementary stress on the network reduces and the swelling of the polymer decreases.

The effect of macromolecules in the surrounding solvent plays an important role at higher concentrations. The degrees of swelling of PE 5 at different gel fractions were predicted from the model at different initial volume fraction of polymer added to the sur-

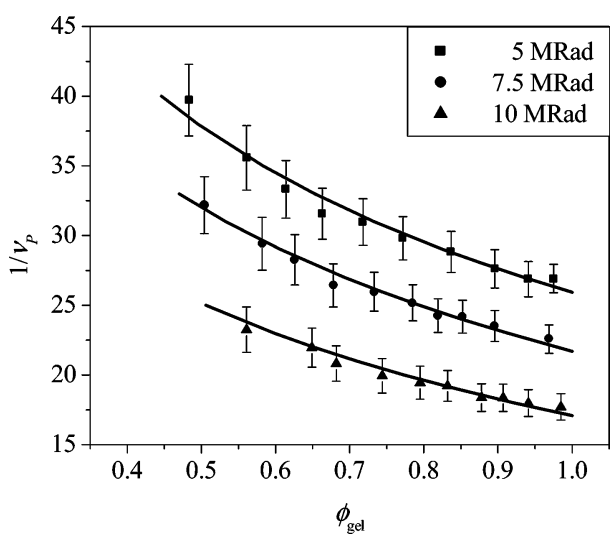

Figure 4. Degree of swelling of partially cross-linked HDPE in $p$-xylene at $100^{\circ} \mathrm{C}$ at different gel fractions. The solid lines are calculated from the model developed for the ternary system, eq 16b. The fraction of active network $\kappa$ was used to fit the model to the experimental results (see Table 1).

rounding solvent prior to swelling (Figure 5). A significant reduction in swelling was observed with the presence of polymer in the surrounding solution. Thus, swelling decreases when the polymer concentration increases in the surrounding bath. This effect becomes insignificant if the swelling is performed with a large excess of solvent and if no additional polymer is added into the surrounding bath beyond the sol macromolecules that originate from the gel. The volume fraction of polymer in the surrounding solution during the abovementioned swelling experiments was of the order of $10^{-4}$. At these small concentrations the effect of the macromolecules in the surrounding bath can be neglected.

The increase in polymer concentration in the bath reduces the difference in chemical potential between the sol macromolecules within the swollen gel and in the surrounding solution. This reduces the loss of sol macromolecules from the specimen. The equilibrium of 


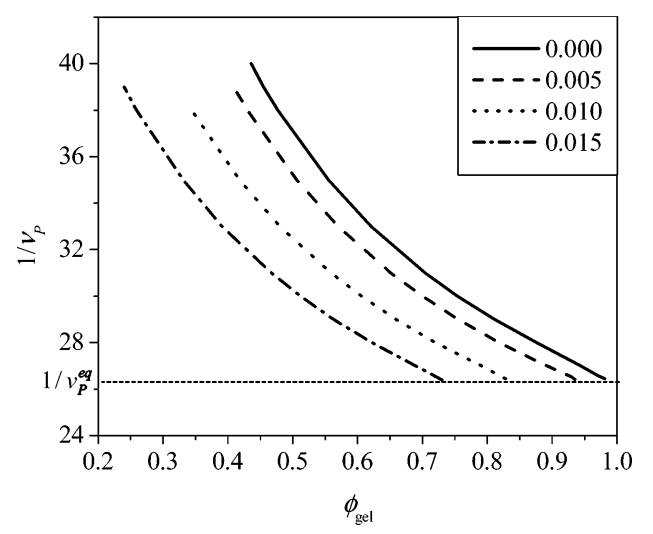

Figure 5. Degree of swelling at different gel fraction for PE 5 predicted from the model at different initial volume fraction of polymer in the surrounding solution. The legend denotes the volume fraction of polymer in the surrounding bath at the beginning of swelling. The dotted line at $1 / v_{\mathrm{P}}^{\mathrm{eq}}$ represents equilibrium swelling where the chemical potentials of both the low molecular weight solvent and the sol macromolecules, inside the swollen gel and outside, are equal to each other, respectively. For PE 5 , we found $1 / v_{\mathrm{P}}^{\mathrm{eq}}=26.4$.

the sol macromolecules within the swollen gel and outside determine the final concentration of sol macromolecules inside the gel. Equation 17b defines the equilibrium state while eq $16 \mathrm{~b}$ at one particular gel fraction determines the final equilibrium. Equations 16b and 17b were solved simultaneously for PE5 (Figure 5) to predict the gel fraction $\phi_{\text {gel }}$ and the degree of swelling $1 / v_{\mathrm{P}}^{\mathrm{eq}}$ at the equilibrium state. The fraction of sol macromolecules retained within the swollen gel increased with increase of initial polymer concentration of the surrounding bath. However, a constant degree of swelling at equilibrium $1 / v_{\mathrm{P}}^{\mathrm{eq}}$ was predicted irrespective of the initial concentration of polymer in the surrounding bath. This phenomenon can be attributed to the following: at equilibrium, the increase in swelling of the polymer network due to the presence of sol macromolecules is invalidated by the decrease in swelling due to the presence of polymer molecules in the surrounding solution. In such a situation, the degree of swelling is equivalent to a binary system of a network swelling in a pure solvent. Also, it is conceivable to increase the polymer concentration in the surrounding solution far enough so that a final equilibrium can be reached without the loss of any sol macromolecules into the surrounding.

The model presented in the paper is a first attempt to predict the swelling behavior of the ternary system. The model predictions mimic the experimental results of the ternary system. However, the swelling process is much more complex than the simplified model considered here. This model can be further refined by including certain phenomena that are neglected here. We presume that branch structure and molecular weight distribution of the sol macromolecules, both within the swollen gel and in the bath, have a significant effect on the time evolution of the swelling. The smaller sol macromolecules are expected to dominate the early diffusion from the sample into the bath. This will result in an increased average molecular weight of the sol macromolecules inside the sample. The polymer solution of the surrounding bath will start out with a molecular weight that is low in the beginning and increases as the experiment evolves. Inside the sample, the diffusion processes also invoke spatial gradients in the sol concentration. Such spatial gradients together with the appropriate properties (diffusivity of both solvents) determine the dynamics of the swelling process. The diffusion of the solvent and sol molecules within the swollen gel can be investigated using pulsed field gradient spin echo NMR spectroscopy, ${ }^{31,32}$ and the effect of the spatial gradient on swelling can be determined.

The effect of the solvents on the elastic free energy of the swollen gel as suggested by Zhao and Eichinger ${ }^{33}$ can also be considered for the ternary model. The standard models for networks such as "Edwards' tube model", 34 "slip tube model", 35 "diffused-constraint model", 36 and "nonaffine tube model" 37 can be incorporated for further modifications of the ternary model and better estimation of the degree of swelling of partially cross-linked polymers.

With the new information from this study, several further experiments can be envisioned. In a modification of the classical experiment in which a network polymer $\left(\phi_{\text {gel }}=1\right)$ is swollen in a low molecular solvent, the solvent bath might be replaced by a polymer solution. The difference in chemical potential of the loose polymer molecules in the bath and within the swollen network will allow the polymer molecules to diffuse into the swollen gel and increase the degree of swelling. Thus, higher degrees of swelling can be obtained by controlling the concentration of polymer in the surrounding bath.

Throughout this study, we neglected the interaction between sol macromolecules and network strands. The model for the ternary system can now be extended to a system where the sol macromolecules are chemically different than the network polymer. This will result in a finite interaction parameter $(\chi \neq 0)$. The effect of the interaction of the sol macromolecules with the network polymer on the degree of swelling will be interesting to analyze.

A further variation to the ternary system would be by tethering of sol macromolecules onto the network. The attachment of the long chain molecules will prevent any loss into the surrounding bath. However, the long tethered molecules are expected to swell the network in similar ways as the sol macromolecules. The sol fraction will remain constant over time eliminating the quasi-steady states.

\section{Conclusions}

The sol macromolecules within the polymer act as an athermal solvent and interact with both the network and the low molecular weight solvent. The model developed for the ternary system predicts that the sol macromolecules within the network generate a substantial increase in the swelling of the polymer. This was also observed in the swelling experiments of HDPE in $p$-xylene where the degree of swelling increased by around $30-40 \%$ due to the presence of $50 \%$ loose chains.

The entropic contribution of the loose chains, which is proportional to the volume fraction of the sol macromolecules, appears as an additional term in the model for the ternary system. Thus, as the loose chains diffuse out of the polymer gel, their contributions toward swelling decreases. The enthalpic interactions of the polymer chains both in the swollen gel and in the surrounding solution appear in the model. However, if swelling is done in a large volume of solvent, the enthalpic and entropic contributions of polymer molecules in the surrounding bath can be neglected for approximate estimation of the degree of swelling. The 
cooperative motion of the network chains is also considered while evaluating the elastic free energy. It is observed that the classical elastic free energy terms are more dominant in the model than the terms appearing due to the cooperative motion of the chains.

Sol macromolecules diffuse out of the polymer specimen into the surrounding bath, thereby increasing the gel fraction. The degree of swelling decreases with time as more and more loose molecules leave the polymer. At any instant of time the swollen polymer is at a quasisteady state when the chemical potential of the solvent within the swollen gel is equal to the chemical potential of the solvent outside. A final equilibrium is reached when the chemical potential of the sol macromolecules within the swollen polymer is equal to the chemical potential of the loose chains outside.

The presence of polymer molecules in the surrounding solution decreases the difference in chemical potential of the macromolecules inside the swollen gel and in the bath. Thus, the degree of swelling decreases with increase in polymer concentration in the surrounding solution. It is possible to reach a state where the polymer concentration is high enough to nullify the effect of sol macromolecules within the swollen gel. In such a situation, the swelling behavior of the partially cross-linked polymer in a solution bath is equivalent to the swelling of a polymer network in a pure solvent. Thus, the degree of swelling attained, at equilibrium, by a partially cross-linked polymer with a certain initial gel fraction is independent of the initial concentration of the polymer solution in the surrounding bath. In the limiting case, where swelling is performed in large excess of solvent, the origin of the polymer in the solution is only from the loss of sol macromolecules; the loose chains present in the outside solution do not have a substantial effect on the degree of swelling.

The model presented here contains many simplifications. Extensions are needed to include various advanced network theories and to predict the times associated with the swelling. It will be important to include effects of the molecular weight distribution of the sol macromolecules, both within the swollen gel and in the bath, and the effect of solvents on the elastic free energy.

Acknowledgment. We thank the National Science Foundation (NSF/CTS-0107156) for funding this project.

\section{References and Notes}

(1) Winter, H. H.; Gappert, G.; Ito, H. Macromolecules 2002, 35, 3325 .
(2) Nandi, S.; Winter, H. H.; Fritz, H. G. Polymer 2004, 45, 4819.

(3) Wall, F. T. J. Chem. Phys. 1942, 10, 132.

(4) Wall, F. T. J. Chem. Phys. 1942, 10, 485.

(5) Wall, F. T. J. Chem. Phys. 1943, 11, 527.

(6) Flory, P. J.; Rehner, J., Jr. J. Chem. Phys. 1944, 12, 412.

(7) Wall, F. T.; Flory, P. J. J. Chem. Phys. 1951, 19, 1435.

(8) James, H. M.; Guth, E. J. Chem. Phys. 1943, 11, 455.

(9) James, H. M.; Guth, E. J. Chem. Phys. 1947, 15, 669.

(10) Gottlieb, M.; Gaylord, R. J. Macromolecules 1984, 17, 2024.

(11) Flory, P. J. In Principles of Polymer Chemistry; Cornell University Press: Ithaca, NY, 1953.

(12) Neuburger, N. A.; Eichinger, B. E. Macromolecules 1988, 21 3060.

(13) Zhao, Y.; Eichinger, B. E. Macromolecules 1992, 25, 6988.

(14) McKenna, G. B.; Crissman, J. M. J. Polym. Sci., Part B: Polym. Phys. 1997, 35, 817.

(15) Chatterji, P. R. Macromolecules 1991, 24, 4214.

(16) McKenna, G. B.; Flynn, K. M.; Chen, Y. Macromolecules 1989, $22,4507$.

(17) Flory, P. J. J. Chem. Phys. 1977, 66, 5720.

(18) Erman, B.; Flory, P. J. J. Chem. Phys. 1978, 68, 5363.

(19) Flory, P. J.; Erman, B. Macromolecules 1982, 15, 800.

(20) Flory, P. J.; Rehner, J., Jr. J. Chem. Phys. 1943, 11, 512.

(21) Flory, P. J.; Rehner, J., Jr. J. Chem. Phys. 1943, 11, 521.

(22) McKenna, G. B.; Flynn, K. M.; Chen, Y. Polymer 1990, 31, 1937.

(23) Gent, A. N.; Tobias, R. H. J. Polym. Sci., Polym. Phys. 1982, 20, 2317.

(24) Russ, T.; Brenn, R.; Geoghegan, M. Macromolecules 2003, 36, 127.

(25) Gaylord, R. J.; Douglas, J. F. Polym. Bull. (Berlin) 1987, 18 , 347. Gaylord, R. J.; Douglas, J. F. Polym. Bull. (Berlin) 1990 23,529 .

(26) Douglas, J. F.; McKenna, G. B. Macromolecules 1993, 26, 3282 .

(27) Structural test methods for determination of gel fraction and swell ratio of cross-linked ethylene plastics. ASTM designation D2765-90, Aug 1990.

(28) Dinzburg, B. N. Elastomers Plastics 1999, 52, 413.

(29) Fetters, L. J.; Lohse, D. J.; Richter, D.; Witten, T. A.; Zirkel, A. Macromolecules 1994, 27, 4639.

(30) Sajkiewicz, P.; Phillips, P. J. J. Polym. Sci., Part A: Polym. Chem. 1995, 33, 853.

(31) Matsukawa, S.; Yasunaga, H.; Zhao, C.; Kuroki, S.; Kurosu, H.; Ando, I. Prog. Polym. Sci. 1999, 24, 995.

(32) Skirda, V. D.; Aslanyan, I. Y.; Philippova, O. E.; Karybiants, N. S. Macromol. Chem. Phys. 1999, 200, 2152.

(33) Zhao, Y.; Eichinger, B. E. Macromolecules 1992, 25, 6996.

(34) Edwards, S. F. Proc. Phys. Soc. London 1967, 92, 9.

(35) Ball, R. C.; Doi, M.; Edwards, S. F.; Warner, M. Polymer 1981, 22, 1010. Edwards, S. F.; Vilgis, T. A. Polymer 1986, 27, 483.

(36) Kloczkoswki, A.; Mark, J.; Erman, B. Macromolecules 1995, 28,5089 .

(37) Rubinstein, M.; Panyukov, S. Macromolecules 1997, 30, 8036. Rubinstein, M.; Panyukov, S. Macromolecules 2002, 35, 6670.

MA048335E 